\title{
Fibroblast-like cells on intraocular lens implants: phagocytosing erythrocytes
}

\author{
J. REIMER WOLTER AND PAUL R. LICHTER
}

From the Departments of Ophthalmology and Pathology of the University of Michigan Hospitals. Ann Arbor, Michigan, USA

SUMMARY An intraocular lens implant had to be removed 4 years after its implantation from the eye of a patient on warfarin (Coumadin) medication because of repeated intraocular haemorrhages. Implant cytology staining revealed a continuous membrane on the removed implant that contained relatively few large foreign body giant cells and great numbers of small fibroblast-like cells. An impressive clean-up function of the fibroblast-like cells with phagocytosis of whole erythrocytes was observed.

Study of 19 intraocular lens implants removed from human eyes at surgery or at autopsy by the 'implant cytology technique' has revealed the presence of cellular membranes on the implants in all cases. ${ }^{1-5}$ These membranes primarily are composed of small star-shaped or bipolar fibroblast-like cells, large foreign body giant cells, and a continuous film-like proteinaceous condensation on the surface of the implant in the spaces between these cells. The small fibroblast-like cells typically show a tendency for accumulation around the giant cells, but they are also seen in the interspaces in a rather regular distribution. In cases with clinically well tolerated implants the giant cells appear to decrease in numbers over the years. However, the fibroblast-like cells are more permanent and remain in the membrane. With time they become more inactive-looking and mostly bipolar in shape, with pigment in their scanty protoplasm.

The origin, nature, and function of the large foreign body giant cells on the surface of lens implants is probably similar to that of foreign body giant cells in the eye under other conditions. Histiocytes are believed to form these cells either by fusion or by nuclear division without protoplasmic separation. Giant cells isolate 'foreign material,' and they have remarkable abilities of phagocytosis and digestion. The term fibroblast-like cells is used as a descriptive name for free-moving macrophages of the anterior eye that have settled on the surface of the foreign

Correspondence to J. Reimer Wolter, MD, Department of Ophthalmology, University of Michigan Hospitals. Ann Arbor, Michigan 48109. USA. substance of intraocular lens implants, developed a bipolar or star-shaped cell body with short branching processes, and probably have an important part in the production of the acellular, film-like, and proteinaceous part of the membranes on implants. The macrophages of the inner eye, unfortunately, have been given many names, and they are also known as phagocytes, free-moving histiocytes, or even microglia, when they enter the eye from the blood vessels of the neuroectodermal tissues of retina or optic nerve. It is the purpose of the present paper to demonstrate the unusual phagocytic abilities of these fibroblast-like cells on the surface of a lens implant that was exposed to repeated haemorrhage.

\section{Case report}

A 70-year-old white female presented with cataracts in June 1978. She had a history of previous pulmonary embolism and had undergone thyroidectomy. She was taking warfarin (Coumadin) and thyroxine. Vision was 20/200 OU due to marked nuclear and posterior subcapsular cataracts. Intraocular pressures by applanation were OD $20 \mathrm{mmHg}$ and OS $17 \mathrm{mmHg}$. The patient desired cataract surgery, with an intraocular lens implant for her left eye. This eye was operated upon under local anaesthesia in July 1978. An extracapsular cataract extraction was done with peripheral iridectomy. During the irrigation of cortical lens material the posterior lens capsule and vitreous face ruptured and an anterior vitrectomy became necessary. A 2-loop Medallion lens implant 641 
through the iris superiorly. The postoperative course was uneventful, but mild iritis and punctate keratopathy were observed postoperatively. There was no evidence of bleeding, and vision could be corrected to $20 / 20$.

In January 1979 the patient had a subconjunctival haemorrhage in the left eye. This was followed by sudden loss of vision due to a vitreous haemorrhage in June 1979. Blood was seen on the iris surface and in the angle, and it was suspected that the bleeding site was anterior rather than posterior. The blood gradually cleared over a 2-week period. Vision again was 20/20. In February 1982 vision in the left eye was again lost suddenly, and another vitreous haemorrhage was diagnosed. Again the blood cleared quickly. However, bleeding episodes recurred often and were associated with increase of intraocular pressure. There was felt to be a possible relationship between this bleeding and the patient's warfarin. This was discontinued by her internist. However, pulmonary embolism occurred 3 weeks later and warfarin was reinstituted. It was noted, that the iris at the pupillary border was slightly eroded by the posts of the lens implant. However, no bleeding site could be discovered. Gonioscopy revealed a grade 4 open angle.

Because of almost continuous repetition of spontaneous bleeding in the left eye it was decided to remove the implant. This was done in July 1982 without any difficulties. It is important to emphasise, that Healon (sodium hyaluronate) was injected to cover the implant before this was pulled from its place in the pupil. Postoperatively much blood in the eye and large areas of choroidal detachment were observed. It was felt that these choroidal detachments were also of a haemorrhagic nature.

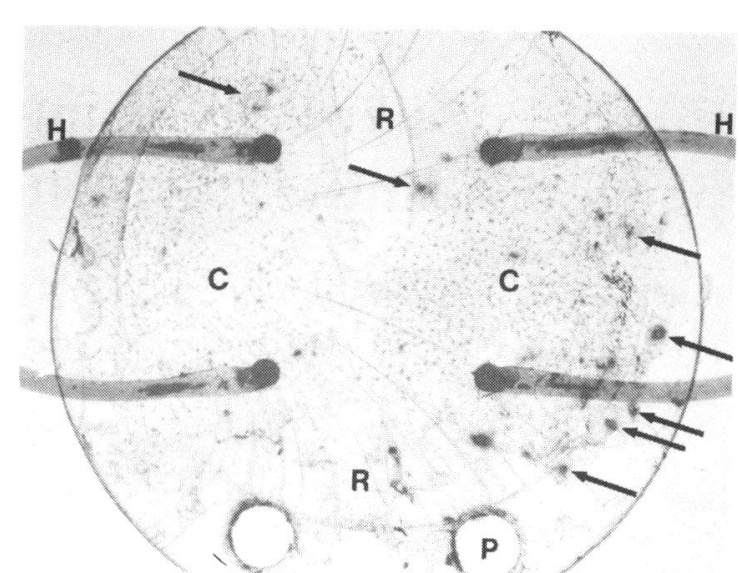

Fig. 1 View of the whole implant-focused on the anterior membrane with 2 perforating suture holes $(\mathrm{P})$ below. Arrows point to some of the larger foreign body giant cells. Two continuous fields of fibroblast-like cells are indicated by $C$. Radiating lines $(\mathrm{R})$ are artificial wrinkles in the plastic surface of the implant from exposure to xylol during the embedding process. (Implant cytology technique, $H$ and E stain, $\times 12$ ).

The implant was dropped into irrigation fluid immediately after its removal and it was transferred to a buffered $10 \%$ solution of formalin within a few minutes. Grossly, the implant was optically clear and transparent. After a few hours of fixation the implant cytology technique was used to stain the cellular membrane on this implant with haematoxylin and eosin. Next this was embedded under a cover glass.

A lower-power view of the implant showed it to be entirely covered by a thin and continuous membrane with great numbers of cells (Fig. 1). Most of these
Fig. 2 A group of fibroblast-like cells surrounding a giant cell $(\mathrm{G})$. Radiating wrinkles in the surface of the implant (R) are an artefact. The insertion of the haptics $(H)$ is seen upper right. (Implant cytology technique, $H$ and $E$ stain, $\times 62$ ).

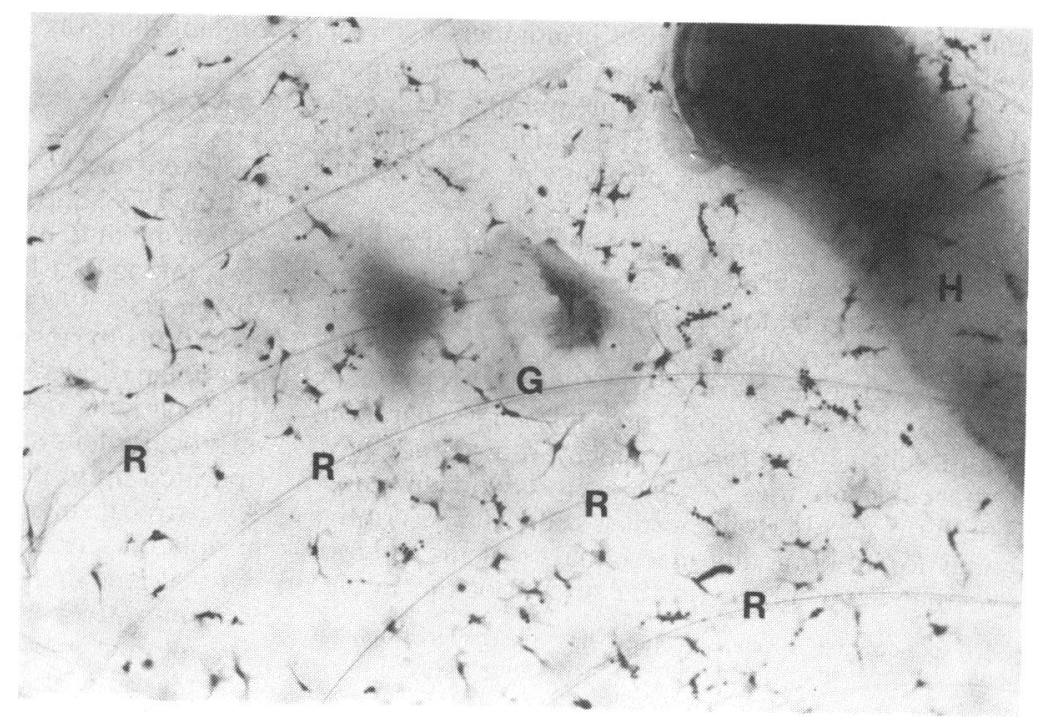




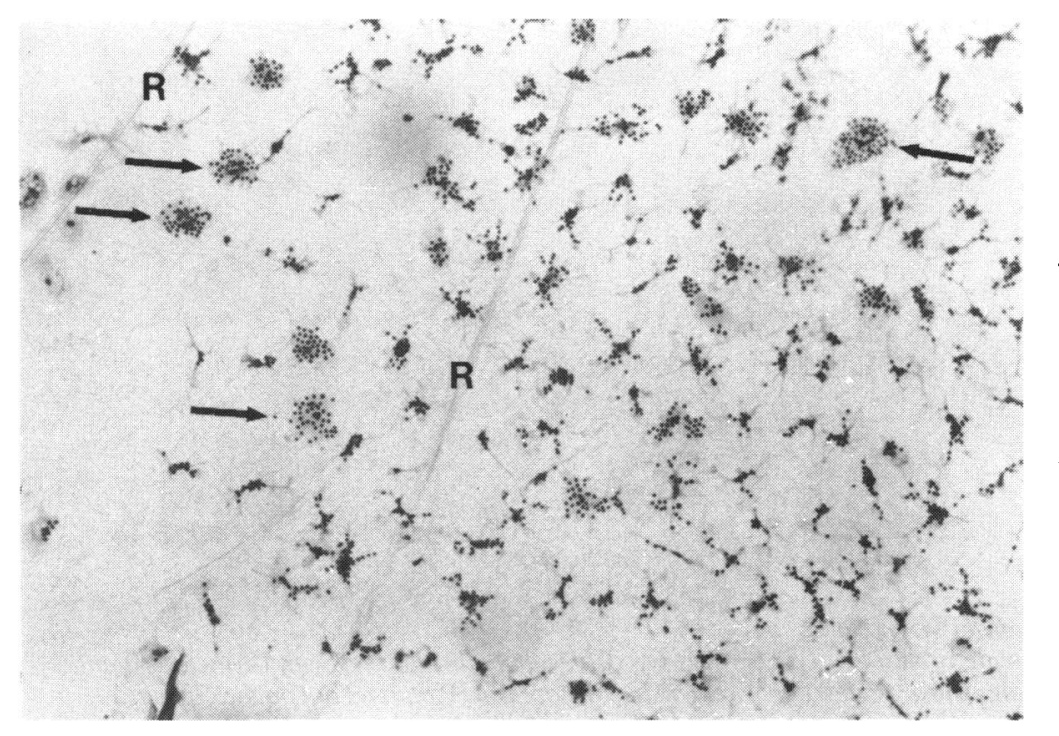

Fig. 3 An area with many star-shaped fibroblast-like cells holding whole erythrocytes with their branching processes and in their protoplasm. Some of these cells have become large and round (arrow) in the process of phagocytosis. R: Radiating wrinkles in the plastic surface. (Implant cytology technique, $H$ and E stain, $\times 62$ ).

were small and arranged in different density on different parts of the implant, but in some larger zones they had a rather regular distribution. Multinucleated giant cells up to about twice the diameter of the haptics were also seen on both surfaces (Fig. 2). They were of irregular distribution. There were about 20 of the large type of these cells on the front of the implant and about 15 on the back.

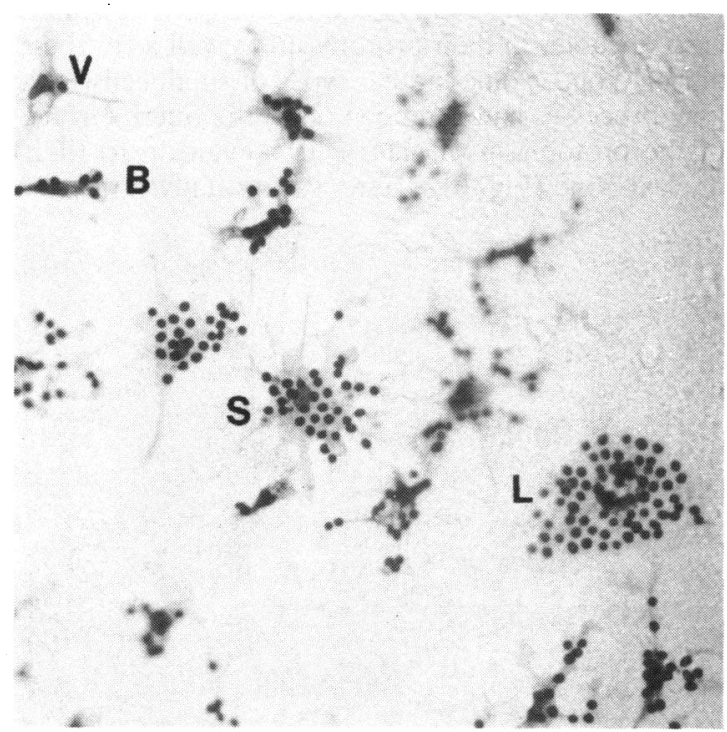

Fig. 4 Details of star-shaped (S), bipolar (B), and large round forms (L) of fibroblast-like cells in the slightly wavy membrane, all with whole erythrocytes on their processes and in their protoplasm. One cell (V) has a clear vacuole and only one whole erythrocyte. (Implant cytology technique. $H$ and $E$ stain, $\times 160$ ).
Some additional giant cells of smaller size were found round the suture holes and on the haptics. Most of the giant cells were seen in marginal zones of the membrane and they were arranged in several groups. Smaller giant cells and large epithelioid cells were seen in a more irregular distribution on both surfaces.

The low-power view (Fig. 1) shows rather regular radiating lines on the surface of the implant. These lines are slight artificial ruptures in the implant surface which develop in the plastic material of some implants during their exposure to xylol during the embedding process.

A film-like membrane of very regular thickness and consistency covering the whole implant was only slightly eosinophilic. It was best recognised in marginal parts of the implant where this had ruptured in the process of surgical removal (Fig. 1). The fact that all parts of the giant cells and small cells were in the plane of this membrane and that no separation from it was recognised indicates that these cells were firmly attached to or part of the membrane.

The small cells were mostly star-shaped, or their main body was also sometimes bipolar. However, all the small cells had numerous delicate processes-all located in the plane of the membrane on the implant. Great numbers of whole erythrocytes of bright red colour were attached to the processes of many of these cells (Figs. 3, 4, and 5). Furthermore, whole erythrocytes were seen in all stages of being incorporated in the protoplasm of these cells. Some of the cells had grown large and round or oval in the process. They contained great numbers of wellpreserved red cells in their central protoplasm (Figs. 4 and 5). The process of erythrocytes being caught and phagocytosed by the small star-shaped or bipolar 


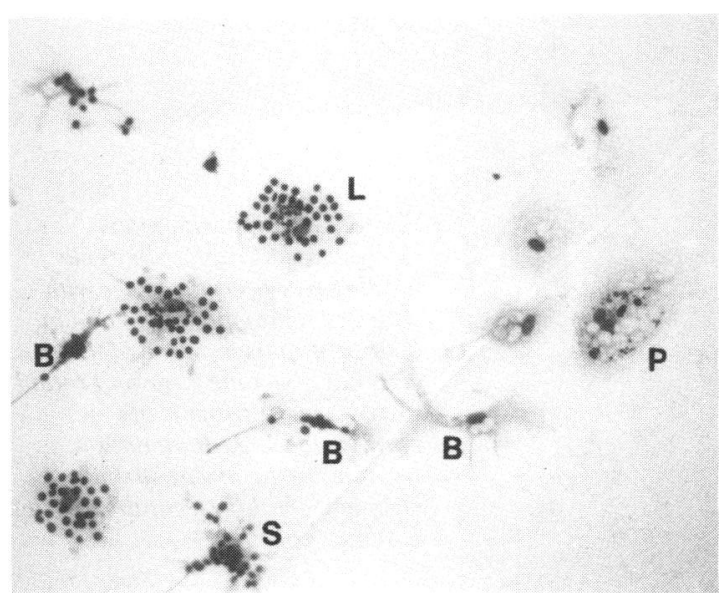

Fig. 5 Details of bipolar (B), star-shaped (S), and large round form $(\mathrm{L})$ of fibroblast-like cells with whole erythrocytes. One round cell contains pigment granules (P) and vacuoles in addition to a few whole erythrocytes. Some of the bipolar and star-shaped cells do not contain erythrocytes or pigment. (Implant cytology technique, $H$ and Estain, $\times 133$ ).

cells was seen in large areas of both the front and the back surfaces of the implant. Not all the small cells were involved with erythrocytes, however, and some of these cells resembled the typical and less active fibroblast-like cells that have been seen on other implants (Figs. 5 and 6). All the small cells had oval and relatively dark-staining nuclei that were distinctly smaller and denser than the round and nucleolated nuclei of the epithelioid and giant cells (Fig. 6). Many of the small cells contained dark brown pigment granules of slightly irregular size (Fig. 5). Some of the

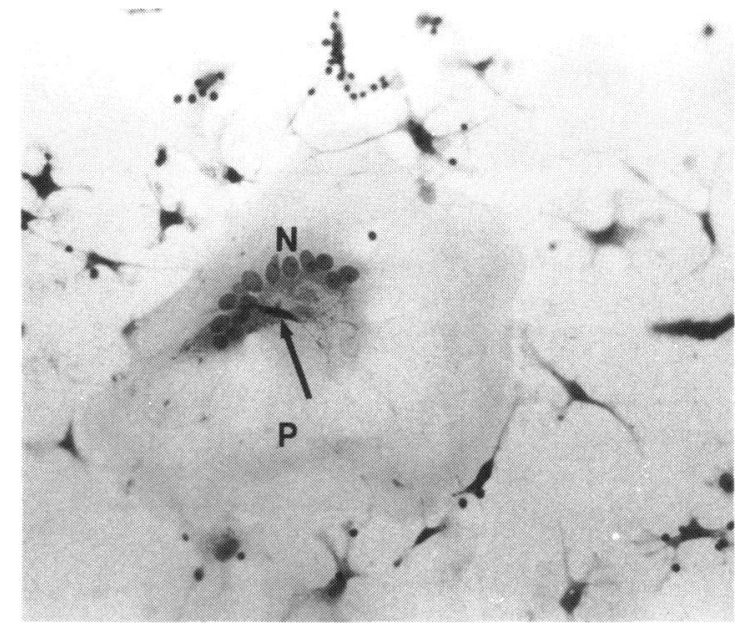

Fig. 6 A foreign body giant cell with 13 nuclei (N) and extensive protoplasm $(\mathrm{P})$ spread out on the implant. Fibroblast-like cells are arranged around the giant cell, and one bipolar cell with long delicate processes is seen on top of the giant cell's nuclear zone (arrow). (Implant cytology technique, $H$ and $E$ stain, $\times 138$ ).

small cells also had single or multiple clear round vacuoles in their protoplasm (Fig. 5).

It is important to emphasise that not a single erythrocyte was found in the protoplasm of a giant cell in this membrane and that the giant cells did not contain much pigment. Some of the giant cells had clear vacuoles in their protoplasm as well as in their nuclear zone. Some bipolar types of small cells with long processes had extended on to the outer surface of the protoplasm of giant cells-even on to their nuclear zone (Fig. 6). There was virtually no extra-
Fig. 7 Loose extracellular erythrocytes diffusely overlying the cellular membrane of the implant extending from a dense accumulation of blood (B) in the upper part of the photograph. Foreign body giant cells in the membrane $(\mathrm{F}$ ) and haptics $(\mathrm{H})$ are seen in the background. (Implant cytology technique, $H$ and $E$ stain, $\times 62$ ).

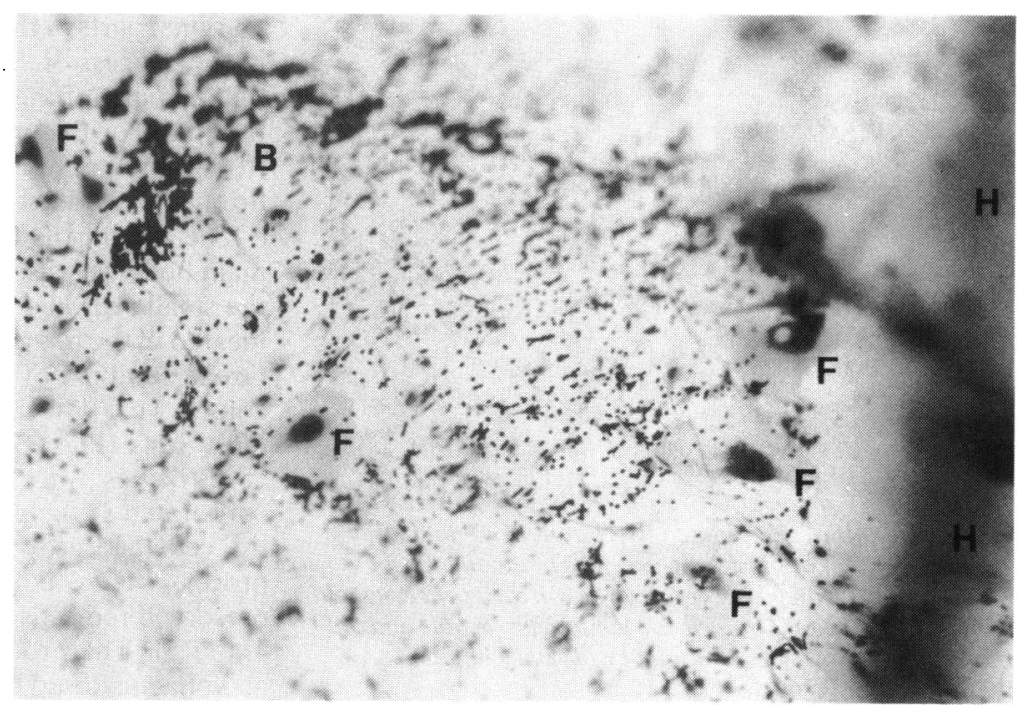


cellular blood directly on the membrane; it was all caught in the processes or contained in the protoplasm of the fibroblast-like cells. However, a larger amount of blood was loosely attached to the anterior surface of the implant, but this was not in direct contact with the membrane (Fig. 7).

\section{Discussion}

Caught in the act of cleaning-up after a haemorrhage, the fibroblast-like cells in the membrane on the lens implant of the present case have revealed a surprising detail of their nature and function. With their long and delicate protoplasmic processes these hungry cells hold on to normal-looking erythrocytes, and they are seen in all stages of incorporating them into their protoplasm. In this process they change from delicate cells with branching processes into rounder elements. However, they remain sessile. Their function is distinctly different from that of common vitreous macrophages, ${ }^{67}$ which phagocytose the remnants of broken-down erythrocytes and deliver these to the next vascular surface of the inner eye or out of the eye through the aqueous humour outflow system. It has been known for a long time that erythrocytes can survive in the vitreous without degeneration for long periods without breaking up or being phagocytosed. ${ }^{8}$ It is therefore surprising to see normal-looking erythrocytes being phagocytosed in great numbers by the fibroblast-like cells on the present lens implant. Haemosiderin granules remaining from the suspected digestion process would at least partly explain the pigmentation of the protoplasm seen in late stages of fibroblast-like cells on lens implants. ${ }^{13}$ However, this breakdown from whole erythrocytes to haemosiderin in the protoplasm of the fibroblast-like cells was not actually seen in the present case. As more removed implants become available for implant cytology study, it will become possible to use an iron stain for differentiation between melanin and haemosiderin pigment in the protoplasm of the late stages of these cells.

The present case again shows that the fibroblastlike cells are a new differentiation of a cell type under the special conditions of lens implantation. They act as quickly available clean-up elements with surface functions. These functions on the implant must have a direct effect on the vision of the involved eye. The large foreign body giant cells in the membrane on lens implants, in contrast, are suspected to be a slow reaction of the inner eye to the substance of the implant ${ }^{4}$-and in the present case they do not at all take part in the clean-up of blood on the implant. It is important to state that perhaps the use of Healon during the surgical removal of the present implant has resulted in the most beautiful preservation of all cells on the surface of the removed implant that we have seen. This would not seem surprising and may actually provide further evidence that Healon in intraocular procedures does what is claimed, namely, protects against damage to delicate surface structures during surgical manipulation.

The cellular membrane found 4 years after implantation on the implant in the present case is similar to membranes that we have seen in other iris-supported lenses. ${ }^{1}$ In number the fibroblast-like cells dominate the cell population, and the relatively few large foreign body giant cells are mainly found in marginal parts of the implant. Small giant cells and epithelioid cells are seen on and next to the haptics and around holes as well as next to the sharp edge of the implant. It is our impression that iris-supported lenses, in principle, have fewer giant cells than anterior chamber lenses. ${ }^{14}$ Cells in membranes of well tolerated implants are arranged as a monolayer. The observation of occasional extension of fibroblastlike cells on to giant cells is in itself a sign of trouble. ${ }^{5}$ The extensive phagocytic activity of fibroblast-like cells is a new observation. The cytological findings as a whole support the clinical impression that the implant had been tolerated as well as this kind of implant is usually tolerated. Study of the implant revealed signs of its exposure to bleeding, but it did not give an indication of the cause of the bleeding.

Use of the implant cytology technique and simple descriptive reporting are starting to give a new dimension to our understanding of the nature and purpose of the cell life on lens implants. We should take the opportunity to study each implant removed during surgery or at autopsy.

This work was supported by the Research to Prevent Blindness Inc., New York, NY.

\section{References}

1 Wolter JR. Cell life on the surface of lens implants. Albrecht von Graefes Arch Klin Ophthalmol 1982; 218: 244-9.

2 Wolter JR. Lens implant cytology. Ophthalmic Surg 1982: 11: 939-42.

3 Wolter JR. Pigment in cellular membranes on intraocular lens implants. Ophthalmic Surg 1982; 13: 726-32.

4 Wolter JR. Foreign body giant cells on intraocular lens implants. Albrecht von Graefes Arch Klin Ophthalmol 1982; 219: 103-11.

5 Wolter JR, Felt DP. Proliferation of fibroblast-like cells in failing intraocular lenses. Ophthalmic Surg 1983; 14: 57-64.

6 Wolter JR. The macrophages of the human vitreous body. Am J Ophthalmol 1960; 49: 99-107.

7 Wolter JR. Leaky cystoid macular edema attracting vitreous macrophages. Ophthalmic Surg 1982; 13: 568-71.

8 Samuels B. Opacities of the vitreous. Arch Ophthalmol 1980; 4: 838-57. 